\title{
Developing and Assessing Educational Games to Enhance Cyber Security Learning in Computer Science
}

\author{
Jinghua Zhang \\ Department of Computer Science \\ Winston-Salem State University \\ Winston-Salem, NC, USA \\ zhangji@wssu.edu \\ Jinsheng $\mathrm{Xu}$ \\ Department of Computer Science \\ North Carolina A\&T State University \\ Greensboro, NC, USA \\ jxu@ncat.edu
}

\author{
Xiaohong Yuan \\ Department of Computer Science \\ North Carolina A\&T State University \\ Greensboro, NC, USA \\ xhyuan@ncat.edu \\ Elva J. Jones \\ Department of Computer Science \\ Winston-Salem State University \\ Winston-Salem, NC, USA \\ jonese@wssu.edu
}

\begin{abstract}
Cyber security is a critical field in Computer Science education today. We need to prepare students for the increasing security challenges they will face in the future. The workforce also needs to have additional cyber security skills to best protect the interests of the nation. Motivating and engaging more students in this field is an important step to meet the needs of future workforce. Games have been successfully used in many areas of education, including Computer Science, to engage students in learning. They provide educational, immersive experiences, which could help underrepresented students gain the confidence to pursue a career or further study in the cyber security field while also inspiring others. The poster will present the three-year project that aims to develop and assess three educational games with in-game assessments to effectively teach cyber security concepts (Access Control, LAN Vulnerabilities and Buffer Overflow). These games will be designed with different levels of difficulty to target students from freshmen to seniors and integrated into the existing computer science curriculum at Winston-Salem State University and North Carolina A\&T State University to benefit a wide range of students. In addition, all these games will be portable, selfcontained and available for download from the project website.
\end{abstract}

Permission to make digital or hard copies of part or all of this work for personal or classroom use is granted without fee provided that copies are not made or distributed for profit or commercial advantage and that copies bear this notice and the full citation on the first page. Copyrights for third-party components of this work must be honored. For all other uses, contact the Owner/Author.

CompEd '19, May 17-19, 2019, Chengdu,Sichuan, China

(C) 2019 Copyright is held by the owner/author(s).

ACM ISBN 978-1-4503-6259-7/19/05.

https://doi.org/10.1145/3300115.3312511

\section{CCS CONCEPTS}

Applied computing $\rightarrow$ Education $\rightarrow$ Computer-assisted instruction

\section{KEYWORDS}

Cyber Security Education, Game-Based Learning, Simulation

ACM Reference format:

Jinghua Zhang, Xiaohong Yuan, Jinsheng $\mathrm{Xu}$ and Elva J. Jones. 2019. Developing and Assessing Educational Games to Enhance Cyber Security Learning in Computer Science. In Proceedings of ACM Global Computing Education conference (CompEd'19). ACM, New York, NY, USA. https://doi.org/10.1145/3300115.3312511 